\title{
African Trypanosomiasis in Young Female in North India - A Rare Case Report
}

\author{
Deepika Parwan ${ }^{1 *}$, Ranjan Kumar ${ }^{1}$ and Sumit Aggarwal ${ }^{2}$ \\ ${ }^{1}$ Department of Pathology, Sarvodaya Hospital and Research Center, Sector 8, Faridabad, Haryana, India \\ ${ }^{2}$ Department of Medicine, Sarvodaya Hospital and Research Center, Sector 8, Faridabad, Haryana, India
}

\begin{abstract}
Human African trypanosomiasis, also known as sleeping sickness, is a vector-borne parasitic disease. It is caused by infection with protozoan parasites belonging to the genus Trypanosoma. They are transmitted to humans by tsetse fly (Glossina genus) bites which have acquired their infection from human beings or from animals harboring human pathogenic parasites. Tsetse flies are found just in sub-Saharan Africa though only certain species transmit the disease. We report a case of human African trypanosomiasis in a 28-year-old Indian female who had a travel history to sub-Saharan Africa, Uganda and she presented with a history of fever, body ache, headache, decreased oral intake, pain lower abdomen, swelling and discharge from forearm chancre since last 4-5 days. Peripheral smear showed heavy parasitemia by flagellated forms of Trypanosoma and the diagnosis of Trypanosoma brucei was given on Peripheral smear report. Serological testing was also done and a diagnosis of West-African trypanosomiasis was confirmed. The patient was successfully treated and made a good recovery. So West-African trypanosomiasis should be considered in the differential diagnosis with presentation of fever with chancre in every person with recent history of travel to African countries as it is universally fatal without treatment.
\end{abstract}

Keywords: Trypanosoma Brucei Rhodesiense, Human African Trypanosomiasis, Female, Sleeping Sickness

\section{Introduction}

Sleeping sickness is an endemic illness of sub-Saharan Africa caused by the flagellate protozoan Trypanosoma brucei and transmitted to human hosts by bites of infected tsetse flies. It occurs in two forms-East African, caused by T. brucei rhodesiense and West African, caused by T. brucei gambiense. Both forms are fatal without treatment ${ }^{[1]}$. The disease usually presents in two stages: afirst or hemolymphatic stage, where the parasite is located in the lymphatic system and blood, and a second or meningoencephalitis stage, which occurs when trypanosomes penetrate the CNS. Although it is uncommon diagnosis in returning travelers attending travel and tropical medicine clinics ${ }^{[2]}$, the number of HAT diagnoses in travelers has been rising in recent years ${ }^{[3]}$ most likely in connection with an increase of tourists visiting endemic areas and improved reporting systems. Our case highlights the significance of taking a travel history from all patients. As international travel becomes increasingly frequent and accessible, rare imported infections may be seen. Trypanosomiasis is relatively easily diagnosed and treated with a high-cure rate. Here we report a case of imported HAT in an Indian female after travelling to Uganda. Written informed consent for publication was obtained from the patient.

\section{Case Report}

We report a case of 28-year-old female who presented with fever, body aches, headache, decreased oral intake, pain in lower abdomen, swelling and discharge from forearm chancre for 4-5 days. After detailed clinical history it was found that she recently travelled to Uganda 10 days back and noticed a rash on her forearm which progressed to a discharging ulcer for which she underwent local dressing and she was admitted to our hospital.

Routine investigations revealed hemoglobin $(\mathrm{Hb}) 12.1 \mathrm{~g} / \mathrm{dl}$, total leucocyte count (TLC) $3.36 \times 10^{3} / \mathrm{ul}$ and platelet count 95000/ul, Rapid Typhoid IgM and Malaria antigen test were negative done by immunochromatography method. Widal test was also negative. Biochemistry parameters were as follows, Serum Sodium $-133 \mathrm{mmol} / \mathrm{L}$, Serum potassium $-3.40 \mathrm{mg} / \mathrm{dl}$, SGOT-47U/L, Prothrombin time 31.7 seconds and INR 2.69. Total bilirubin $-1.33 \mathrm{mg} / \mathrm{dl}$, Direct bilirubin $-0.57 \mathrm{mg} / \mathrm{dl}$ and Indirect bilirubin- $0.76 \mathrm{mg} /$ dl. Ultrasound abdomen was done and found to be normal and Chest $\mathrm{X}$ ray was also found to be normal. On peripheral smear examination, heavy parasitemia was seen with morphological appearance of flagellate protozoan [Figure 1 and Figure 2], suggestive of Trypanosoma and sample were also sent for further confirmation. In view of multiorgan involvement and heavy parasitemia, she was referred to a higher Centre for further treatment by Suramin.

\section{Discussion}

Human trypanosomiasis in endemic in Africa as sleeping sickness (caused by Trypanosoma brucei) and Latin 


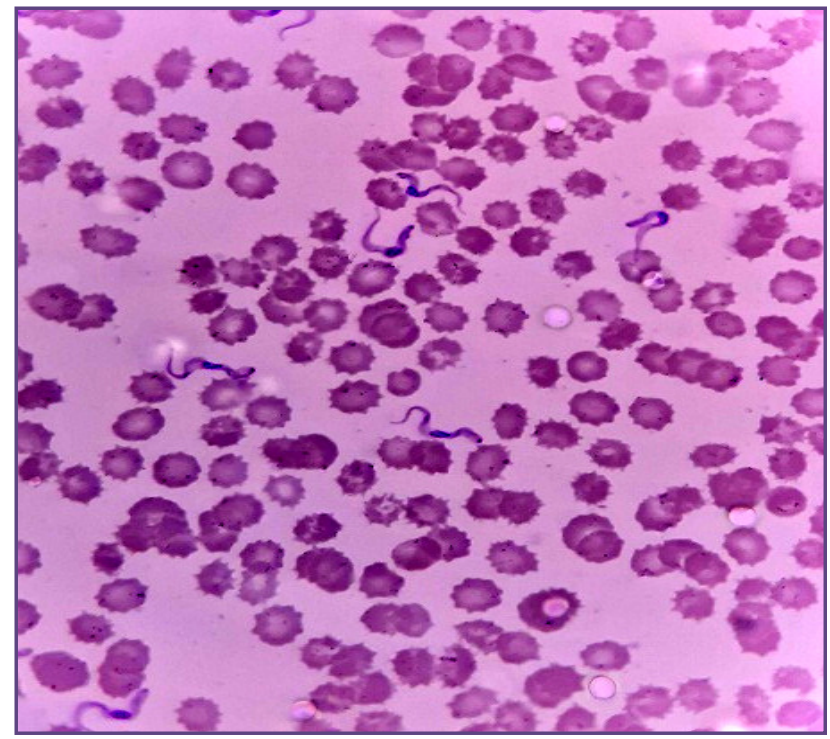

Fig. 1: Leishman-stained peripheral blood smear showing heavy parasitemia by flagellate Trypanosoma (100X magnification).

American as Chaga's disease (caused by Trypanosoma cruzi). Human trypanosomiasis is not known to exist in India. However, animal trypanosomiasis is endemic in India and is caused by Trypanosoma evansi in cattle/horses or Trypanosoma lewisi in rats ${ }^{[4]}$. In our case patient had recent travel history to Uganda after which she developed the symptoms, which can be considered as Imported case. The patient represents the first reported case of imported Human African Trypanosomiasis in Delhi-NCR, of African trypanosomiasis, who had history of travel to sub-Saharan African country.

Bryan et $\mathrm{al}^{[5]}$ also reviewed 15 cases in United States of East African trypanosomiasis, found that the most common manifestations were fever, rash, lethargy, headache, gastrointestinal complaints, confusion, and myalgia. Other authors have described lymphadenopathy, hepatomegaly, splenomegaly, signs of renal dysfunction, and (rarely) cardiopulmonary decompensation. In our case also patient presented with fever, body ache, abdominal pain and chancre on forearm but no organomegaly was found.

A classic case of HAT goes through clinically distinct stages starting with development of a chancre at the site of the tsetse fly bite accompanied by regional lymphadenopathy. As the disease progresses from local symptoms to the first stage and causes significant morbidity while mortality may ensue even with appropriate therapy a generalized infection, fever is one of the commonest, albeit nonspecific sign. The second stage of the disease is associated with chronic encephalopathy associated with headache and

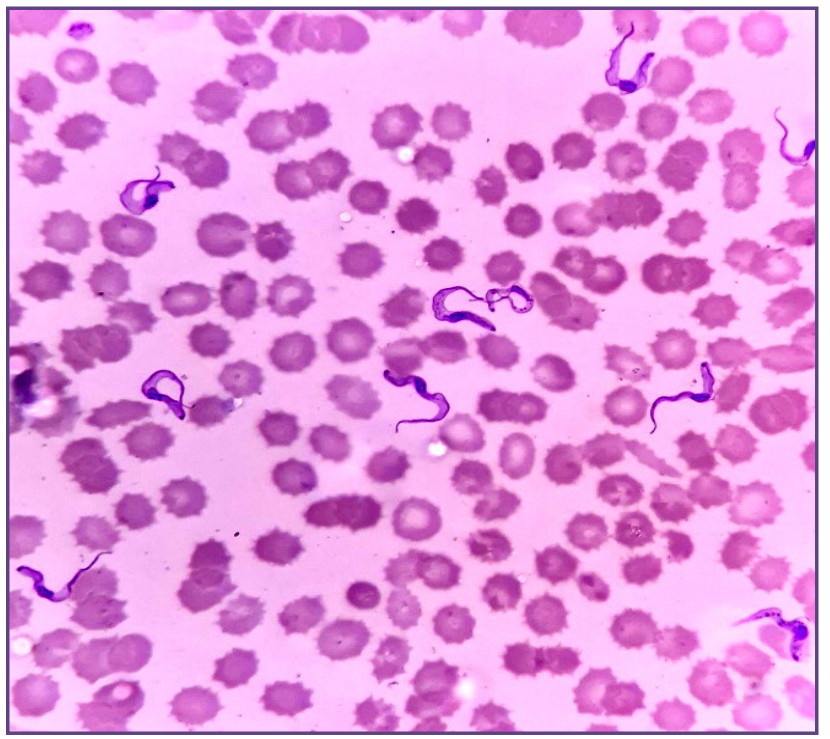

Fig. 2: Leishman-stained peripheral smear show flagella undulating membrane and the central round nucleus (100x magnification).

mental changes ${ }^{[6]}$ Untreated, rhodesiense HAT is uniformly fatal, and causes significant morbidity while mortality may ensue even with appropriate therapy ${ }^{[7]}$. In Our case also patient presented with these symptoms and presentation including fever, headache and presenting with chancer at forearm which get unnoticed and patient was given wound dressing. But after correct diagnosis, patient was shifted to higher Centre where proper treatment was given and patient recovered fully.

\section{Conclusion}

To conclude this case, it highlights the importance of taking proper travel history from patient which in our case was neglected initially and therefore classical chancre on forearm of the patient was missed, presenting with the symptoms of fever, headache, abdominal pain and presence of chancer which could have given probable differential diagnosis towards the very rare case of Human African trypanosomiasis. Therefore, it also signifies about how important is the role of peripheral blood examination by an expert pathologist since this is a rare disease in India and the delay in diagnosis is fatal and to my best knowledge this is the only case reported in Delhi-NCR which can be categorized as imported disease.

Consent: Written informed consent was taken from patient for the publication of this case report and all the images. Thank you

\section{Acknowledgements}

Nil 


\section{Funding}

Nil

\section{Competing Interests}

There is no conflict of interest

\section{Reference}

1. World health organization, Human African trypanosomiasis (sleeping sickness) epidemiological update, wkly Epidemiol Rec 2006;8:71-80.

2. Human African trypanosomiasis in a Spanish traveler returning from Tanzania; PLoS NegL Trop Dis 11(3): e0005324

3. SchlagenhaufP, WeldL, Goorhuis A, Gautret P, Weber R, Von Sonnenburg Fetal. Travel associated infection presenting in Europe (2008-12): an analysis of Euro travelnet longitudinal, surveillance data and evaluation of the effect of the pre- travel consultation. Lancet infect diseases .2015;15(1):5564. Epub2014/2016

4. Joshi PP. Human trypanosomiasis in India: is it an emerging new zoonosis? In: Munjal YP, Editor, API Textbook of Medicine. 9thedition. New Delhi:Jaypee Brothers; 2013.p.10-13.

5. Bryan RT, Waskin HA, Richards FO, Bailey TM, Juranek DD. African trypanosomiasis in American travelers: A twenty-year review. In: Stef- fen R, Lobel HO, Haworth J, Bradley DJ, eds. Travel medicine: proceedings of the First Conference on International Travel Medicine. Berlin: Springer-Verlag, 1988:384 - 8.

6. Stich A, Abel PM, Krishna S (2002) Human African trypanosomiasis. The reemergence of sleeping sickness presents a major public health problem. BMJ 325: 203-206.

7. Meltzer E, Leshem E, Steinlauf S, Michaeli S, Sidi Y, et al. (2012) Short Report: Human African Trypanosomiasis in a Traveler: Diagnostic Pitfalls. Am J Trop Med Hyg 87: 264-266.

*Corresponding author:

Deepika Parwan, Department of Pathology, Sarvodaya Hospital and Research Center, Sector 8, Faridabad, Haryana, India Phone: +91 99711811080

Email: deepikaparwan@gmail.com

Date of Submission : 14/12/2020

Date of Final Revision : 11/03/2021

Financial or other Competing Interests: None.

Date of Acceptance : 09/04/2021

Date of Publication : 30/04/2021 